\title{
Factores culturales y emocionales de los pacientes diabéticos frente a la enfermedad
}

\author{
Cultural and emotional factors of diabetic patients facing the disease
}

María Cecilia Galimberti Oliveira « 1,a,b, Justina Isabel Prado Juscamaita 1,c, José Bernardino Gutarra Vara 1,c

Filiación y grado académico

Universidad Nacional Hermilio Valdizán, Huánuco, Perú.

a Magister en Antropologia Medica y Salud Internacional.

Magister en Desarrollo Social.

Doctor en Ciencias de la Salud.

(10. ORCID iD de María Galimberti

https://orcid.org/0000-0001-8966-402X

(1). ORCID iD de Justina Prado

https://orcid.org/0000-0002-6558-4233

(1) ORCID iD de José Gutarra

https://orcid.org/0000-0001-7329-2700

Contribución de los autores

MCGO: trabajo de campo y entrevista, análisis

e interpretación de los datos y redacción del artículo científico.

JIPJ: marco teórico, análisis e interpretación de los datos y redacción del artículo científico.

JBGV: análisis del contenido y redacción del artículo científico.

Fuentes de financiamiento

La investigación fue desarrollada en el marco de las actividades de investigación docente con una subvención básica de la Universidad Nacional Hermilio Valdizán de Huánuco, Perú.

Conflictos de interés

Ninguno.

Recibido: 12-05-2020

Arbitrado por pares

Aceptado: 05-08-2020

Citar como

Galimberti M, Prado J, Gutarra J. Factores culturales y emocionales de los pacientes diabéticos frente a la enfermedad. Rev Peru Cienc Salud. 2020; 2(3):153-60. doi: http://doi.org/10.37711/ rpcs.2020.2.3.193

\section{RESUMEN}

Objetivo. Conocer los factores culturales y emocionales de pacientes diabéticos frente a la enfermedad. Métodos. La metodología fue cualitativa, con diseño etnográfico. La muestra fue 10 pacientes, con diabetes tipo 2, trabajadores de la Universidad Nacional Hermilio Valdizán de Huánuco a quienes se aplicó la guía de entrevista semi-estructurada, el análisis de datos se realizó con el programa Atlas.ti. Resultados. El paciente diabético muestra, diferencias culturales que incluyen al sujeto y su experiencia de enfermedad. La enfermedad limita la vida social del paciente. Los valores importantes encontrados se asientan fundamentalmente en el apoyo familiar y en sentirse productivos asumiendo responsabilidades. Sus representaciones culturales no concuerdan con sus prácticas culturales ya que, en teoría, ellos saben lo que deben hacer para adherirse al tratamiento (realizar dieta, hacer ejercicios y tomar medicamentos) pero en la práctica hacen lo contrario. Los pacientes diabéticos están influidos en su conducta por el illness o padecimiento: los cuales constituyen un conjunto de emociones, sentimientos y experiencias que afectan la salud del paciente. Ellos asocian la enfermedad a las emociones y sentimientos que experimentan en su vida social, estas emociones las cuales transforman su cuerpo y su vida. Las situaciones críticas o impresiones fuertes que experimentan, aumentarán su presión arterial y también los niveles de glicemia. Conclusión. Los factores culturales del paciente diabético, sus formas de pensar y actuar, están determinados por sus condiciones de vida y por una serie de factores: socioculturales, económicos y emocionales.

Palabras clave: humanos, diabetes mellitus tipo 2, presión arterial, glucosa en sangre, condiciones sociales, análisis de los datos, emociones, determinación de la presión arterial, comportamiento social, ejercicio, dieta (Fuente: DeCS - BIREME).

\section{ABSTRACT}

Objective. To know cultural and emotional factors of diabetic patients facing the disease. Methods. Methodology was qualitative, with ethnographic design. Sample comprised 10 patients, with type 2 diabetes, workers of Universidad Nacional Hermilio Valdizán de Huánuco to whom a semi-structured interview was applied, and data analysis was carried out with the program Atlas.ti. Results. The diabetic patient shows cultural differences that include the subject and their experience of illness. Illness limits patient's social life. The important values found are settled fundamentally in family support and in feeling productive assuming responsibilities. Their cultural representations do not match their cultural practices since, in theory, they know what to do to adhere to treatment (diet, exercise, and take medicine) but in practice they do the opposite. Diabetic patients are influenced by their behavior due to the illness or condition: It constitutes a set of emotions, feelings and experiences that affect the patient's health. They associate the disease with the emotions and feelings they experience in their social life. These emotions transform your body and your life. The critical situations or strong impressions they experience will increase their blood pressure and also blood sugar levels. Conclusion. The cultural patterns of the diabetic patient, their ways of thinking and acts are determined by their living conditions and by a series of factors: sociocultural, economic and emotional.

Keywords: Humans, Diabetes Mellitus Type 2, Blood Pressure, Blood Glucose, Social Conditions, Data Analysis, Emotions, Blood Pressure Determination, Social Behavior, Exercise, Diet (Source: MeSH - NLM). 


\section{INTRODUCCIÓN}

Las poblaciones tienen sus propias representaciones mentales y modelos culturales sobre la salud, no siempre coincidentes con el discurso sanitario, que guían sus acciones de modo determinante. La mera constatación empírica de los hechos sociales se ha mostrado insuficiente para entender y ayudar a transformar los comportamientos humanos siendo, en este sentido, las cuestiones ideológicas y afectivas las que cobran una importancia relevante. La investigación estructural, mediante el uso de técnicas cualitativas, nos permite acercarnos al conocimiento de estas cuestiones, orientando las actuaciones de la educación sanitaria y promoción de la salud ${ }^{(1)}$.

La diabetes mellitus (DM) es, según la Organización Mundial de la Salud ${ }^{(2)}$, una amenaza mundial. Se calcula que en el mundo existen más de 180 millones de personas con diabetes, siendo probable que esta cifra aumente al doble para el 2030. Esta se constituye como una de las enfermedades no trasmisibles (ENT) de salud más frecuentes e importantes entre el 2 y el $5 \%$ de la población mundial por la carga de enfermedad en términos de discapacidad y mortalidad prematura que, en su evolución, va comprometiendo a diferentes órganos afectando la calidad de vida de quienes la padecen. En este sentido, la diabetes es considerada, dentro de las condiciones diabéticas crónicas, una de las más demandantes en términos físicos, psicológicos y emocionales. Inicialmente las personas diagnosticadas con diabetes pasan por un proceso de ajuste que puede generar reacciones y sentimientos tales como: coraje, depresión, ansiedad, frustración y pérdida de valor por la vida ${ }^{(3)}$.

El síndrome metabólico, la obesidad, la hipertensión arterial y la dislipemia son las patologías más frecuentes que conducen al desarrollo de la diabetes mellitus 2 (DM 2). La evidencia que demuestra que el control de estos factores de riesgo evita o retrasa el desarrollo de la enfermedad es clara. En este sentido, diversas intervenciones sobre el estilo de vida a través de cambios de alimentación, actividad física y programas de educación nutricional, revelan una disminución en la progresión de prediabetes a DM2 entre un 50 y $60 \%{ }^{(4)}$.

Las enfermedades crónicas son vividas y reinterpretadas por los individuos que las sufren, convirtiéndose en padecimientos illness como señala Fitzpatrick (1990). "la enfermedad significa también más tiempo en la que puede reinterpretar la significación de sus síntomas y su tratamiento" ${ }^{(5)}$. Una característica común de los hombres con DM2 es la negación de la enfermedad al considerarse incurable. Esta, además del tratamiento farmacológico, requiere un estilo de vida saludable que exige cambios en los patrones culturales de la familia. El problema no termina ahí, Leininger afirma: "la cultura puntualiza lo que es salud, enfermedad, vida, muerte; igualmente la necesidad de sentir atención, o sentir ira, tristeza o melancolía" (6).

Frente a la enfermedad pueden aparecer también creencias que subestiman y desacreditan el impacto de la DM, ("la diabetes no es nada"), o la asocian a ultranza con sus "complicaciones" (ceguera, amputación e insuficiencia renal). El primer enfoque aparece muy relacionado con el desconocimiento de las formas de expresión de la enfermedad o al hecho de que sus manifestaciones más visibles aparecen a largo plazo; lo cual conlleva a subvalorar los cuidados de salud. La segunda alternativa sobredimensiona los aspectos más negativos de la enfermedad y desconoce o minimiza el valor de los cuidados de la salud, la adherencia al tratamiento y la educación en DM como pilares fundamentales para el control metabólico. Por ello conduce a respuestas de temor, sensación de falta de control y vulnerabilidad, que pueden expresarse en la "paralización" del uso de recursos de afrontamiento activo de la enfermedad ${ }^{(7)}$.

La desigualdad de género podría ser la expresión de otras exposiciones de riesgo para la salud derivadas de las dobles cargas de trabajo (en el hogar, con el cuidado de los niños y los mayores, más el resto de trabajos domésticos y fuera de aquel, la subordinación en la toma de desiciones, la mayor precariedad laboral y el menor apoyo social para sus cuidados personales, especialmente en las mujeres que viven solas ${ }^{(8)}$. Existen algunos estudios en los cuales se comenta lo anterior, demostrando el alto impacto en la estabilidad de esta enfermedad, pues parece estar estrechamente vinculado a la situación social, cultural y económica ${ }^{(9)}$.

Los padecimientos crónicos repercuten sensiblemente en el bienestar y la calidad de vida de quienes lo padecen y sus allegados. Su solo diagnostico implica la pérdida del estado de salud, ademas de la puesta en marcha de cuidados y nuevas rutinas que permitan seguir las instrucciones del equipo de salud. En el caso de los diabéticos se han informado de mayores niveles de depresión, hasta seis veces más alta, que el resto de la poblacion: ansiedad, baja autoestima y sentimientos de desesperanza y minusvalía ${ }^{(10)}$.

La prevalencia de diabetes en las Américas varía entre el 10 y $15 \%$; en el Perú se estima en un 5,5\%. La magnitud de la misma está en aumento, debido al incremento de factores como la obesidad, el sobrepeso, fundamentalmente la obesidad abdominal ${ }^{(11)}$, así como el sedentarismo y los hábitos inadecuados de alimentación. 
En los últimos años la DM2 es considerada como una enfermedad multifactorial en la que influyen aspectos como el cambio en los hábitos alimenticios, sedentarismo y una esperanza de vida mayor ${ }^{(12)}$. En el Perú, la mayoría de los pacientes no presentan un buen control glucémico; por lo cual las complicaciones crónicas han aumentado en frecuencia, provocando limitaciones funcionales a corto y largo plazo, deteriorando la vida física, psicológica, social y familiar de los que la padecen, causando así preocupación a nivel de los servicios médicos, por el consumo de recursos, sin llegar a los objetivos glucémicos esperando así como la repercusión de los miembros, tanto familiares, amigos y otras personas capaces de aportar un apoyo en la vida del paciente diabético para llegar al mantenimiento glucémico adecuado. La DM representa un serio problema de salud. El aumento de su incidencia y prevalencia, su carácter complejo y la severidad de los impactos económicos, sanitarios y psicosociales que genera, contribuyen a la importancia de reflexionar sobre sus efectos desde el espacio individual y social más inmediato a las personas que viven con esta enfermedad ${ }^{(13)(14)}$.

Por otro lado, desde el enfoque cultural, ser un humano es llegar a ser un individuo, orientado por esquemas culturales, por sistemas de significación históricamente creados en virtud de los cuales formamos, ordenamos, sustentamos y dirigimos nuestras vidas ${ }^{(15)}$.

La cultura determina una visión particular del mundo para cada grupo o colectivo humano que, a diferencia de la científica, se cimientan en una base filosófica estructurada y lógica que explica muchas de las concepciones, significados, valores y creencias de un pueblo. De modo que estas prácticas de los cuidados culturales tienden a estar arraigadas e influyen en el cuidado y tratamiento de la enfermedad ${ }^{(16,17)}$. Por ello nos interesó estudiar los factores culturales y emocionales de pacientes diabéticos frente a la enfermedad; debido a que los estilos de vida como el ejercicio y la dieta influyen en el estar sano o enfermo, así como los factores emocionales: situaciones críticas o conflictivas, stress, depresión o tristeza afectan a la salud del paciente. El número de personas con DM está creciendo rápidamente en nuestro país ${ }^{(18)}$ y la causa principal de su veloz incremento es el importante cambio en el estilo de vida de la población peruana, caracterizada por una ingesta excesiva de alimentos de alto contenido calórico como la "comida chatarra" y las bebidas azucaradas, así como una reducción de la actividad física que conllevan a altas tasas de sobrepeso y obesidad ${ }^{(19)}$.

La causalidad de la DM se encuentra conformada por un conjunto de elementos relacionados entre sí que llevan a identificar al padecimiento como una enfermedad que invade la vida de la persona en sus diversas esferas. Los estilos de vida inadecuados, como es la alimentación insuficiente o inadecuada; la población considera además los estados emocionales alterados y las impresiones fuertes (sustos, corajes y otros) son los factores de riesgos ${ }^{(20)}$. En este contexto, este estudio se justifica porque proporciona una comprensión sociocultural del funcionamiento de la enfermedad crónica para conocer y concientizar a la población sobre la importancia de asumir la enfermedad, adaptándose a convivir con ella sin que ello signifique un costo psicológico. Por ende el objetivo del estudio fue conocer los factores culturales y emocionales de los pacientes diabéticos frente a la enfermedad.

\section{MÉTODOS}

\section{Nivel, tipo y diseño de investigación}

El tipo de estudio cualitativo, de naturaleza etnográfica, empleado nos ha permitido recoger el punto de vista del paciente diabético, así como su narrativa, experiencias y subjetividad, teniendo en cuenta el contexto. Según Garrido ${ }^{(21)}$, quien resalta el método de Spradley cuyo enfoque etnográfico está preocupado por el significado de las acciones y eventos que atribuyen los actores sociales, algunos significados están directamente expresados en el lenguaje y otros son sólo comunicados indirectamente a través de la palabra y acción. Las personas en cualquier sociedad usan estos complejos sistemas de significados para organizar su comportamiento, comprenderse a sí mismas, a los demás, y dar sentido al mundo. Estos sistemas de trabajo construyen su cultura y la etnografía permite generar la teoría de la cultura que, a su vez, busca crear y comprobar la hipótesis para posibilitar otras investigaciones.

\section{Diseño de la investigación}

El enfoque del estudio fue cualitativo ${ }^{(22)}$ para describir, explicar e interpretar los factores culturales y emocionales del paciente diabético frente a su enfermedad, teniendo en cuenta el contexto.

La población estuvo conformada por todos los docentes y administrativos diabéticos de la Universidad Nacional Hermilio Valdizan (UNHEVAL) de Huánuco, Perú. La muestra estuvo conformada por 10 pacientes con DM seleccionados al azar y por saturación que cumplieron con los criterios de inclusión y exclusión:

\section{Criterios de inclusión}

- Pacientes con diagnóstico de DM2.

- Trabajadores administrativos y docentes de la UNHEVAL.

- Edad entre 20 y 70años de edad. 
- Pacientes que firmaron el consentimiento informado.

\section{Criterios de exclusión:}

- Pacientes con DM1

- Trabajadores administrativos y docentes que no trabajan en la UNHEVAL

- Pacientes que no firmaron el consentimiento informado

\section{Métodos, técnicas e instrumentos de recolección de datos}

La técnica utilizada fue la entrevista semiestructurada relacionada a las variables del estudio: los factores culturales y emocionales en pacientes diabético. El instrumento utilizado fue el cuestionario, donde el paciente contestó un conjunto de preguntas relacionadas a las variables mencionadas ${ }^{(23)}$.

\section{Análisis de datos}

El análisis cualitativo de los datos recolectados, trascritos a texto, consistió en la sistematización de la información, codificada para explicar y analizar. El análisis cualitativo fue interactivo y recurrente con ayuda del programa Atlas ti.

\section{Rigor ético de la investigación}

Durante el desarrollo de la investigación se consideraron los principios éticos en la interacción con los informantes tales como su autorización y la confidencialidad requerida, así como el consentimiento informado.

\section{RESULTADOS}

\section{Estilos de vida en pacientes diabéticos en relación al cuerpo, el ejercicio y la dieta.}

Respecto a la dimensión sobre cómo funciona el cuerpo, se encontró que 7 pacientes presentan: "sed se le nubla la vista y presenta bajo peso"; 2 manifiestan "estaba mareada, no recordaba por donde estaba caminando y tenía nauseas"; 1 describe: "hormigueo en cara y cabeza como mariposas". o"la visión le ha disminuido".

Sobre el funcionamiento del cuerpo con la enfermedad 7 manifiestan: "mi cuerpo está normal, pero tengo alteraciones cuando reniego, me sube la glucosa, mi cuerpo tiembla y me da ansiedad", "no debo preocuparme porque me sube la glucosa. Mi diabetes es emotiva debido a que yo he garantizado a mi compadre un préstamo de 10 mil soles, le voy a cobrar y no tiene plata. Por lo tanto, yo debo pagar al banco 890 soles mensuales. Esa es la principal causa de mi enfermedad y de mi pie diabético, la deuda me enferma". Sobre la dieta el $10 \%$ (1) manifiesta que "Al comienzo, la nutricionista casi me mata; debía comer la mitad de un pan, sin sal y poca comida. Yo hice lo contrario para no morir. Justo una enfermera me preguntó si yo quería vivir. Yo le dije que sí. Entonces me dijo que tenía que comer"10 \% (1) refiere: "no me siento mal, pero existen ciertas limitaciones; ya no puedes comer lo de siempre, debes ser metódico en la alimentación y obligadamente hacer ejercicios. Pero como tengo una hernia en la columna no puedo hacer ejercicios".

Tabla 1. Factores emocionales y estado de ánimo frente a la enfermedad en pacientes diabéticos

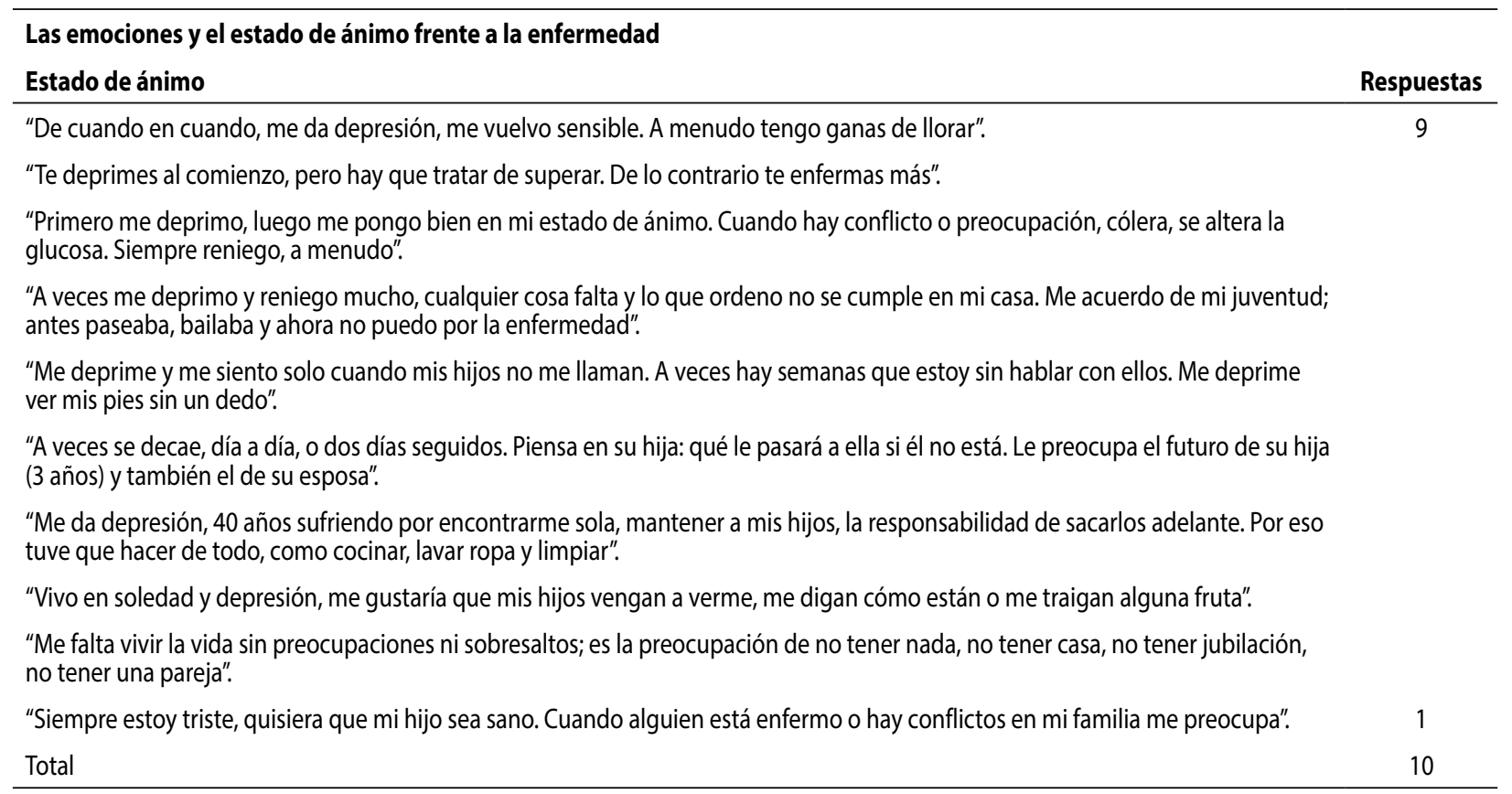

Fuente. Entrevista a informantes claves por saturación. 
Tabla 2. Tratamiento de la enfermedad y factores emocionales frente a la enfermedad

\begin{tabular}{|c|c|}
\hline \multicolumn{2}{|l|}{ Tratamiento de la enfermedad y emociones frente a la enfermedad } \\
\hline Tratamiento & Respuestas \\
\hline metformina, divenclamida. & 4 \\
\hline $\begin{array}{l}\text { metformina y culen, sacha-culandro,perejil y metformina insulina. } \\
\text { yacon,berenjena, agua, pasuchaca, cindilla, Livenclamida y Metformina }\end{array}$ & 5 \\
\hline Piña en extracto con pepinillo licuado en ayunas y tilo. & 1 \\
\hline Total & 10 \\
\hline No está satisfecho, los médicos te regañan. Tendrían que explicarte más sobre la enfermedad. & 2 \\
\hline \multicolumn{2}{|c|}{$\begin{array}{l}\text { No está satisfecho, los médicos cuando te atienden no te dicen la verdad, les pides algo y no te dan. No hacen más de lo que } \\
\text { deben hacer. No te dicen lo que debes hacer, no toman compromiso y no te hablan claro. }\end{array}$} \\
\hline Sí, pero no asiste a sus controles, ella (el) se controlan solos. & 2 \\
\hline Sí & 6 \\
\hline Total & 10 \\
\hline No, la enfermedad es parte de él. No piensa en la enfermedad, si piensas te aniquilas más rápido. & 2 \\
\hline Sí, por las complicaciones de algún órgano. & 8 \\
\hline \multicolumn{2}{|l|}{ Estamos preocupados de no terminar en diálisis o con la pierna amputada. } \\
\hline \multicolumn{2}{|l|}{ Tengo temor a que mi cuerpo cambie. } \\
\hline \multicolumn{2}{|l|}{ Tengo miedo a la muerte, dejar a mis hijos que quiero. No hay como la luz del día. } \\
\hline \multicolumn{2}{|l|}{ Tengo miedo que avance la enfermedad y quien va a ver a mis hijos. Una mamá es una mamá. } \\
\hline Total & 10 \\
\hline
\end{tabular}

Fuente. Entrevista a informantes claves por saturación.

En cuanto a estilos de vida en relación a como asumen la enfermedad, como la viven y si cuentan con el apoyo familiar en pacientes diabéticos.

Apreciamos que 7 pacientes refirieron que hacen su vida con normalidad con la enfermedad; textualmente dicen: sí, puedo seguir estudiando y capacitándome para tener más oportunidades, sí solo con limitaciones, no bebo, no como carbohidratos, sí, hace su vida con normalidad. El aspecto psicológico es importante. En la práctica hay que asumir la enfermedad, el problema es cuando lo niegas". De otra parte, 10 pacientes refieren contar con el apoyo de su familia, con: "la esposa, esposo, hija, hijos, familia, madre, hermano".

\section{Factores emocionales sobre las expectativas de enfermedad en pacientes diabéticos.}

Al respecto, 4 pacientes refirieron expectativas de la enfermedad: "espero que haya una vacuna o una cura para la enfermedad". "espero que haya cura para la diabetes; se trata de morir con dignidad sin que te amputen las piernas o sin que estés dializado". Por otro lado, 3 pacientes manifiestan: "la enfermedad depende de uno mismo. De acuerdo a la calidad de vida que uno tiene, la enfermedad será llevadera. No hay cura para la diabetes, todo depende de uno". "La Diabetes no tiene cura; por tanto se debe ser metódico en la alimentación y cumplir las prescripciones del médico. Por los años

Tabla 3. Factores emocionales en relación a la ansiedad en pacientes diabéticos

Respuestas

\section{Ansiedad}

Sí "Ansiedad, sí, el trabajo como jefe hace estar ansioso. Antes salía a la hora, ahora no tiene tiempo; trabaja más de sus horas
establecidas y le genera estrés y ansiedad".
"Tengo ansiedad con la enfermedad, no sabes que sucederá con tu cuerpo. Tengo temor que me amputen las piernas".
No
Total

Fuente. Entrevista a informantes claves por saturación. 
Tabla 4. Los estilos de vida y las relaciones sociales en pacientes diabéticos

\begin{tabular}{ll}
\hline & Respuesta \\
\hline Pertenece a algún grupo social & 5 \\
Sí & 5 \\
No & \\
Desarrolla alguna actividad social & 4 \\
Sí & 6 \\
No & \\
Asiste a fiestas y compromisos sociales & 6 \\
Sí & 4 \\
No & \\
Tiene amigos y los frecuenta & 7 \\
Sí & 4 \\
- Sale con ellos hacer deporte, a veces a tomar. & 3 \\
- Asiste a fiestas y discotecas & 3 \\
No & \\
\hline Fuente. Entrevista a informantes claves por saturación. &
\end{tabular}

que tengo he aprendido a manejar las situaciones. No hay que darle importancia a la enfermedad". Por último 3 pacientes comentaron: "que dicen no comprometa a otros órganos". "Estar sin un pie es estar inutilizado". "Más allá ellos (mis hijos) no van a poder a hacer nada". ¿Qué haría sin un pie? No podría ir a mis citas.

\section{DISCUSIÓN}

En diversos estudios se define el estilo de vida como un factor de riesgo para la incidencia de DM2 en la población, con detrimento de su calidad de vida; y se establece la necesidad de la modificación de los estilos de vida para el control de la enfermedad y la conservación de la calidad de vida. En este sentido, se recomienda implementar programas educativos para ellos ${ }^{(18)}$. Sin embargo, se desconoce qué estilos de vida se ven afectados en una persona posterior al haberse diagnosticado con DM2 y su efecto en los diferentes dominios y facetas de la calidad de vida. Por otro lado, los estilos de vida son parte importante de los factores socioculturales. Al respecto Galindo y Padilla ${ }^{(24)}$ indican que los factores socioculturales como experiencias vitales, experiencias laborales, religión, costumbres y tradiciones, prácticas curativas y ritos, señalados en la teoría general del déficit de autocuidado, muestran un efecto positivo sobre la capacidad de autocuidado de las personas con DM2. De este modo, se requiere desarrollar programas comprensivos de promoción y atención al paciente con diabetes que incorporen la cultura del enfermo mediante el uso sistemático de disciplinas tales como la psicología social, la antropología, la sociología, la lingüística y la historia y poder así establecer puentes socioculturales entre pacientes y proveedores de servicios de salud ${ }^{(25)}$.
Una reciente revisión sistémica de individuos en riesgo con prediabetes mostró que las intervenciones en el estilo de vida recomendadas para prevención y tratamiento son el principal objetivo para controlar la diabetes Sin embargo, en la sociedad hay grandes impedimentos con relación a las estrategias que pueden desarrollar estilos de vida saludables. Por ejemplo, la alimentación saludable es una parte importante en el gerenciamiento de la diabetes, así como el acceso a programas que promueven el ejercicio físico. Sin embargo, en todo el mundo hay escasez en el suministro de frutas y vegetales con base en una ingestión recomendada de por lo menos cinco porciones diarias, superior al $58 \%$ en países de baja renta.

Por consiguiente, en la presente investigación hemos podido apreciar que los factores culturales y emocionales influyen en la conducta del paciente, en su relación social con el entorno natural y social, en su relación con los "otros", en el tipo de alimentación que debe consumir, en los ejercicios que debe realizar, así como la adherencia al tratamiento. Un paciente diabético es un paciente sensible y se verá afectado por el cambio en sus emociones; experimentará miedo, temor, incertidumbre, ansiedad, depresión y un cúmulo de emociones y sentimientos que lo volverán cada vez más vulnerable: "que haría sin un pie, no podría ir a mis citas". Estos resultados son congruentes a los encontrados por Rivas et al ${ }^{(3)}$ quienes en su estudio han reportado deterioros en el aspecto emocional del paciente diabético con niveles altos de ansiedad y depresión. Por otro lado González et al.(.27) afirman que la enfermedad crónica genera angustia, miedo, culpa, desesperanza y tiene sus propias peculiaridades biológicas, ya que como cada individuo y familia tienen sus propias interpretaciones y significados. Una investigación cualitativa nos permite acercarnos al conocimiento de estas cuestiones, orientando las actuaciones de la educación sanitaria y promoción de la salud ${ }^{(1)}$.

\section{CONCLUSIONES}

\section{Factores culturales}

Los patrones culturales del paciente diabético, sus formas de pensar y actuar, están determinados por sus condiciones de vida y por una serie de factores socioculturales, económicos y emocionales. La enfermedad limita la vida social de los pacientes, son más retraídos, su mundo social se circunscribe a su vida familiar y las actividades laborales. Cuando asisten a un compromiso, las prohibiciones de no poder comer o tomar algo, hace que eviten este tipo de reuniones. Los valores importantes para los pacientes diabéticos consisten en contar con el apoyo familiar y sentirse productivos asumiendo 
responsabilidades, desempeñando alguna actividad y sintiéndose útiles a los demás.

La mayoría de pacientes entrevistados saben lo que deben hacer para adherirse al tratamiento; reconocen que deben hacer revisiones periódicas para el control de la glicemia, dieta y realizar ejercicios, como parte del tratamiento biomédico. Estas normas prescriptivas forman parte de sus representaciones culturales; sin embargo, en sus prácticas culturales, al paciente diabético le cuesta hacer dieta y realizar ejercicios. La dieta y el ejercicio no es parte de su forma de vida. Como estas normas son impuestas por el médico, tienen un carácter de obligatoriedad y, por lo tanto, no siempre serán cumplidas, ya que en los patrones culturales del paciente se cree que la dieta cuesta más que la comida o que esta es un castigo. Parte de su sistema de vida consiste en tener hábitos de higiene y algunas prácticas de relajación para contrarrestar el estrés y, por lo tanto, la enfermedad.

En sus estilos de vida, los pacientes reconocen que no consumen alimentos dulces ni salados, evitan el consumo de grasa y de alcohol. Algo muy importante es que evitan renegar porque consideran que si reniegan la glicemia subirá y agravará la enfermedad. En su mayoría, los pacientes mencionaron que no hacen ejercicio. La mayoría de pacientes tienen como expectativa estar sanos, controlar la enfermedad y evitar que esta comprometa otros órganos. Para ellos es importante controlar sus emociones durante la enfermedad, para que esta no avance y así tener amigos en la enfermedad.

\section{Factores emocionales}

Los pacientes diabéticos están influidos en su conducta por el illness o padecimiento: un conjunto de emociones y sentimientos que afectan la salud del paciente. Ellos asocian la enfermedad a las emociones y sentimientos que experimentan en su vida social y estas afectan y modifican su vida; experimentando: miedo, tristeza, desconfianza, depresión, ansiedad, estrés, cólera. Las impresiones fuertes que experimentan aumentarán su presión arterial y también los niveles de glicemia. Este cúmulo de subjetividad creará un contexto social caracterizado por el aislamiento social, baja autoestima, desconfianza, desconfianza en la enfermedad y desconfianza en el futuro.

Las creencias están dadas por un conjunto de reglas y por aquello que es significativo para el paciente diabético. Por lo tanto, ellos creen en su propia medicina como el uso de la herbolaria a través de infusiones para contrarrestar la diabetes; así mismo, creen en una fuerza sobrenatural denominada Dios quien trata, cura y salva al paciente cuando está a punto de morir. La felicidad para los pacientes diabéticos es relativa, temporal, instantánea, de momentos: "hoy estás feliz y mañana triste". Es no tener ningún mal y que todas las cosas que hagas te salgan con éxito; ellos son felices viendo a su familia feliz.

Finalmente se recomienda incorporar la perspectiva sociocultural para el estudio y tratamiento del paciente diabético, teniendo en cuenta su cultura y condiciones de vida. Así mismo es importante contar con la participación de un antropólogo médico dentro del equipo de profesionales de salud con la finalidad de incluir las variables socioculturales de la enfermedad y recoger la perspectiva del sujeto y sus experiencias, para lograr una mejor adherencia al tratamiento y mejorar la relación médico-paciente.

\section{REFERENCIAS BIBLIOGRÁFICAS}

1. Gil M, Estrada C, Pires ML, Aguirre R. La investigación cualitativa y la promoción de la salud en la Comunidad de Madrid. Rev. Esp. Salud Publica [Internet] 2002 Oct; 76(5): 451-459 [Consultado 2020 Sep 18] Disponible en: http://scielo.isciii.es/scielo.php?script=sci_arttext\&pi$\mathrm{d}=\mathrm{S} 1135-57272002000500007 \& \operatorname{lng}=\mathrm{es}$

2. Velázquez-Monroy O, Grupo Ensa 2000, et al. Prevalencia e interrelación de enfermedades crónicas no transmisibles y factores de riesgo cardiovascular en México: Resultados finales de la Encuesta Nacional de Salud (ENSA) 2000. Arch. Cardiol. Méx [Internet] 2003; 73(1): 62-77 [Consultado 2020 Sep 19] Disponible en: http:// www.scielo.org.mx/scielo.php?script=sci_arttext\&pi$\mathrm{d}=\mathrm{S} 1405-99402003000100009$

3. Rivas-Acuña V, García-Barjau H, Cruz-León A, Morales-Ramón F, Enríquez-Martínez RM, Román-Álvarez J. Prevalencia de ansiedad y depresión en las personas con diabetes mellitus tipo 2. Salud en Tabasco [Internet] 2011; 17(1-2): 30-35 [Consultado 2020 Sep 17] Disponible en: https://www.redalyc.org/articulo.oa?id=48721182005

4. Hernández M, Batlle MA, Martínez B, San-Cristóbal R, Pérez-Díez $S$, Navas-Carretero $S$, et al. Cambios alimentarios y de estilo de vida como estrategia en la prevención del síndrome metabólico y la diabetes mellitus tipo 2: hitos y perspectivas. Anales Sis San Navarra [Internet] 2016 Ago; 39( 2 ): 269-289 [Consultado 2020 Sep 19] Disponible en: http://scielo.isciii.es/scielo.php?script=sci_arttext\&pid=S1137-66272016000200009\&lng=es

5. Campos-Navarro R, Torrez D, Arganis-Juárez EN. Las representaciones del padecer en ancianos con enfermedades crónicas. Un estudio en la Ciudad de México. Cad. Saúde Pública [Internet] 2002 Oct; 18(5): 1271-1279 [Consultado 2020 Sep 18] Disponible en: http://www.scielo.br/scielo.php?script=sci_arttext\&pid=S0102-311X2002000500019\&lng=en. https://doi. org/10.1590/S0102-311X2002000500019

6. García-Reza C, Cruz E, Gómez D, Toxqui MJG, Sosa BC. La percepción de un grupo de hombres sobre la Diabetes Mellitus: contribuciones a la enfermería. Esc. Anna Nery [Internet] 2014 Dec; 18(4): 562-569 [Consultado 2020 Sep 
18] Disponible en: http://www.scielo.br/scielo.php?scrip$\mathrm{t}=$ sci_arttext\&pid=S1414-81452014000400562\&Ing=en

7. Ledón L. Impacto psicosocial de la diabetes mellitus, experiencias, significados y respuestas a la enfermedad. Rev Cubana Endocrinol [Internet] 2012 Abr; 23(1): 76-97 [Consultado 2020 Sep 18] Disponible en: http://scielo.sld.cu/scielo.php?script=sci_arttext\&pi$d=S 1561-29532012000100007 \&$ Ing=es

8. Escolar A. Determinantes sociales frente a estilos de vida en la diabetes mellitus de tipo 2 en Andalucía: ¿la dificultad para llegar a fin de mes o la obesidad? Gac Sanit [Internet] 2009 Oct; 23(5): 427-432 [Consultado 2020 Sep 19] Disponible en: http://scielo.isciii.es/scielo.php?scrip$\mathrm{t}=\mathrm{sci}$ _arttext\&pid=S0213-91112009000500012\&lng=es

9. Prada DM, Hernández C, Gómez JA, Gil R, Reyes Y, Solís U, et al. Evaluación de la calidad de vida relacionada con la salud en pacientes con artritis reumatoide en el Centro de Reumatología. Rev Cuba Reumatol [Internet] 2015 Abr; 17(1): 48-60 [Consultado 2020 Sep 18] Disponible en: http://scielo.sld.cu/scielo.php?script=sci_arttext\&pi$\mathrm{d}=\mathrm{S} 1817-59962015000100008$ \&lng $=e s$

10. Riveros A, Cortazar-Palapa J, Alcazar FL, Sanchez-Sosal JJ. Efectos de una intervención cognitivo-conductual en la calidad de vida, ansiedad, depresión y condición médica de pacientes diabéticos e hipertensos esenciales. International Journal of Clinical and Health. 2005; 5(3): 445-462.

11. Carrillo-Larco RM, Bernabé-Ortiz A. Diabetes mellitus tipo 2 en Perú: una revisión sistemática sobre la prevalencia e incidencia en población general. Rev. perú. med. exp. salud publica [Internet] 2019 Ene; 36(1): 2636 [Consultado 2020 Sep 18] Disponible en: http:// www.scielo.org.pe/scielo.php?script=sci_arttext\&pi$d=S 1726-46342019000100005 \& \operatorname{lng}=e s$

12. Pérez $A$, Berenguer $M$. Algunas consideraciones sobre la diabetes mellitus y su control en el nivel primario de salud. MEDISAN [Internet] 2015 Mar; 19(3): 375-390 [Consultado 2020 Sep 18] Disponible en: http://scielo.sld.cu/scielo.php?script=sci_arttext\&pi$\mathrm{d}=$ S1029-30192015000300011\&lng=es

13. Ledón L. Impacto psicosocial de la diabetes mellitus, experiencias, significados y respuestas a la enfermedad. Rev Cubana Endocrinol [Internet] 2012 Abr; 23(1): 76-97 [Consultado 2020 Sep 18] Disponible en: http://scielo.sld.cu/scielo.php?script=sci_arttext\&pi$d=$ S1561-29532012000100007\&lng=es

14. Organización Mundial de la Salud. Informe Mundial sobre la Diabetes: Resumen de orientación [Internet] [Consultado 2020 Sep 17] Disponible en: https://www.who. int/diabetes/global-report/es/\#

15. García OH. La cultura humana y su interpretación desde la perspectiva de la cultura organizacional. Pensamiento \& Gestión [Internet] 2007; 22: 143-167 [Consultado 2020 Sep 18] Recuperado de: https://www.redalyc.org/articulo.oa?id=64602204

16. Cruz-León A, Luna-Victoria M, Flor M. Cultura y cuidado en la gestación: Una aproximación a la atención intercultural prenatal. Salud en Tabasco [Internet] 2014; 20(2): 63-68 [Consultado 2020 Sep 15] Recuperado de: https://www.redalyc.org/pdf/487/48735406007.pdf

17. López C, Ávalos MI. Diabetes mellitus hacia una perspectiva social. Rev Cubana Salud Pública [Internet] 2013 Jun; 39(2): 331-345 [Consultado 2020 Sep 18] Disponible en: http://scielo.sld.cu/scielo.php?script=sci_arttext\&pi$\mathrm{d}=$ S0864-34662013000200013\&lng=es
18. Noda JR, Pérez JE, Málaga G, Aphang MR. Conocimientos sobre su enfermedad en pacientes con diabetes mellitus tipo 2 que acuden a hospitales generales. Rev Med Hered [Internet] 2008 Abr; 19(2): 46-47 [Consultado 2020 Sep 18] Disponible en: http:// www.scielo.org.pe/scielo.php?script=sci_arttext\&pi$d=S 1018-130 X 2008000200005 \& \operatorname{lng}=e s$

19. Ministerio de Salud. Guía de práctica clínica para el diagnóstico, tratamiento y control de la Diabetes Mellitus tipo 2 en el primer nivel de atención. Dirección General de Intervenciones Estratégicas en Salud Pública. Lima: MINSA; 2016.

20. López C, Ávalos MI. Diabetes mellitus hacia una perspectiva social. Rev Cubana Salud Pública [Internet] 2013 Jun; 39(2): 331-345 [Consultado 2020 Sep 18] Disponible en: http://scielo.sld.cu/scielo.php?script=sci_arttext\&pi$\mathrm{d}=$ S0864-34662013000200013\&lng=es

21. Garrido N. EL Método de James Spradley en la investigación cualitativa. Enfermería (Montevideo) [Internet] 2017 Oct; 6(spe): 37-42 [Consultado 2020 Sep 18] Disponible en: http://www.scielo.edu.uy/scielo.php?script=sci_arttext\&pid=S2393-66062017000200037\&lng=es. http://dx.doi.org/10.22235/ech.v6iespecial.1449

22. Méndez V, Becerril V, Morales M, Pérez VM. Autocuidado de las adultas mayores con diabetes mellitus inscritas en el programa de enfermedades crónicas en Temoaya, México. Cienc. Enferm. [Internet] 2010 Dic; 16(3): 103-109 [Consultado 2020 Sep 18] Disponible en: https://scielo.conicyt.cl/scielo.php?script=sci_arttext\&pi$\mathrm{d}=$ S0717-95532010000300011\&lng=es.

23. López-Carmona JM, Ariza-Andraca CR, Rodríguez-Moctezuma JR, Munguía-Miranda C. Construcción y validación inicial de un instrumento para medir el estilo de vida en pacientes con diabetes mellitus tipo 2. Salud pública Méx [Internet] 2003 Ago; 45(4): 259267 [Consultado 2020 Sep 18] Disponible en: http:// www.scielo.org.mx/scielo.php?script=sci_arttext\&pid=S0036-36342003000400004\&lng=es

24. Galindo-Martínez MG, Rico-Herrera L, Padilla-Raygoza N. Efecto de los factores socioculturales en la capacidad de autocuidado del paciente hospitalizado con diabetes tipo 2. Aquichan [Internet] 2014 Mar; 14(1): 7-19 [Consultado 2020 Sep 18] Disponible en: http:// www.scielo.org.co/scielo.php?script=sci_arttext\&pi$\mathrm{d}=$ S1657-59972014000100002\&lng=en. http://dx.doi. org/10.5294/aqui.2014.14.1.1

25. García JE, Salcedo AL, López B. Una aproximación al conocimiento cultural de la diabetes mellitus tipo 2 en el occidente de México. Desacatos. Revista de Ciencias Sociales [Internet] 2006; (21): 97-108 [Consultado 2020 Sep 18] Recuperado de: https://www.redalyc.org/articulo.oa?id=13902107

26. Rivas-Acuña V, García-Barjau H, Cruz-León A, Morales-Ramón F, Enríquez-Martínez RM, Román-Álvarez J. Prevalencia de ansiedad y depresión en las personas con diabetes mellitus tipo 2. Salud en Tabasco [Internet] 2011; 17(1-2): 30-35 [Consultado 2020 Sep 17] Recuperado de: https://www.redalyc.org/articulo.oa?id=48721182005

27. González N, Tinoco AM, Benhumea LE. Salud mental y emociones en pacientes con enfermedades crónico-degenerativas. Un acercamiento a la diabetes mellitus tipo 2. Espacios Públicos [Internet] 2011; 14(32): 258-279 [Consultado 2020 Sep 18] Recuperado de: https://www. redalyc.org/articulo.oa?id=67621319013 